\title{
CAN GALVANIC VESTIBULAR STIMULATION REDUCE SIMULATOR ADAPTATION SYNDROME?
}

\author{
Rebecca J. Reed-Jones, ${ }^{1}$ James G. Reed-Jones, ${ }^{2}$ Lana M. Trick, ${ }^{2}$ Lori A. Vallis ${ }^{1}$ \\ ${ }^{1}$ Department of Human Health and Nutritional Sciences \\ ${ }^{2}$ Department of Psychology \\ University of Guelph \\ Guelph, Ontario, Canada \\ E-mail: rreed@uoguelph.ca
}

\begin{abstract}
Summary: Electrical stimulation of the vestibular sensory system during virtual environment simulations has been proposed as a method to reduce the incidence of simulator adaptation syndrome (SAS). However, there is limited empirical evidence to support this hypothesis. It is especially important to provide vestibular stimulation in driving simulators because an absence of vestibular cues may alter driver behaviour and reduce vehicle control. This study examined the application of galvanic vestibular stimulation (GVS) as a technique to reduce symptoms of SAS and improve vehicular control in a fixed-based driving simulator. Nineteen participants drove two visually distinct virtual environments (high and low visual cues). In addition, each of these worlds was experienced with and without GVS. Post-drive scores on the Simulator Sickness Questionnaire (SSQ) were used to evaluate the effect of GVS on SAS. In addition, three driving variables were measured to examine driving performance: steering variability, lane departures, and average vehicular speed. GVS application while driving resulted in significant decreases in total SSQ and disorientation symptoms. Greater vehicular control was also observed (as shown by reduced steering variability) when GVS was used in combination with visual cues along the simulated edge of the road. These results support that GVS may be used in fixed-base driving simulators to create vestibular motion cues and reduce SAS.
\end{abstract}

\section{INTRODUCTION}

Driving simulators permit driver performance to be assessed under controlled conditions, and are especially important when measuring behaviours or situations that might produce collision if tested in the real world. However, when in a driving simulator some individuals experience Simulator Adaptation Syndrome (SAS), which may include one or more of the following symptoms: nausea, disorientation, dizziness, headache, and/or difficulty focusing. Sometimes the symptoms are so severe that the experiments have to be terminated and data is lost (Stanney, Mourant \& Kennedy, 1998). Thus, simulator adaptation syndrome is one of the leading methodological problems in driving simulation research.

Little is known about the underlying mechanisms that induce SAS. SAS is especially prevalent in studies that involve static (fixed-base) driving simulators, which are often chosen because they are less expensive than dynamic (moving-base) simulators. One theory is that SAS occurs due to the visual perception of self motion, induced by the virtual environment (VE), conflicting with the perception of a static situation from the vestibular system (Flanagan, May \& Dobie, 2004). 
As such, it has been suggested that the use of galvanic vestibular stimulation (GVS) while driving in a VE may reduce the incidence of SAS (Kemeny \& Panerai, 2003). GVS is a technique that has been widely used as a tool to elicit a vestibular perception of roll motion (Fitzpatrick \& Day, 2004). GVS involves application of an electrical current via electrodes placed bilaterally over the mastoid process, providing direct stimulation of the eighth cranial nerve afferent. This provides a vestibular perception of lateral acceleration of approximately $2 \%$ $\mathrm{s}^{2}$ (Fitzpatrick \& Day, 2004). Thus, providing a vestibular motion stimulus through GVS may help reduce the conflict between the perception of a static (vestibular) and a perception of a dynamic (visual) situation, resulting in reductions in SAS in fixed-base simulators (Kemeny \& Panerai, 2003). Furthermore, the use of GVS during simulated driving may produce a more realistic experience of lateral motion during turns. However, to date, there is limited empirical evidence to support the contention that application of GVS during VE simulations reduces SAS. Furthermore, to our knowledge there has never been an investigation that evaluated the effects of GVS on simulator sickness scores as measured by the Simulator Sickness Questionnaire (SSQ; Kennedy, Lane, Berbaum \& Lilental, 1993), the current standard for evaluating SAS in simulator research.

Normally, in real-life driving, lateral acceleration is perceived by the vestibular apparatus (primarily the semi-circular canals of the ear) when drivers negotiate a curve on a road. This information is used to estimate and adjust vehicular speed, ensuring adequate control of the vehicle in the curve (Reymond, Kemeny, Droulez \& Berthoz, 2001). However, Reymond et al. (2001) found that when drivers negotiated turns in a fixed-base driving simulator, their pattern of speed reduction differed from that seen in actual driving and while driving in a motion-base simulator. In addition, participants reported greater difficulty in handling the vehicle while driving curves in the fixed-base simulator. These results suggest that when drivers negotiate a curve, visual cues alone are insufficient to regulate the required velocity changes. Therefore, drivers may negotiate curves faster in fixed-base driving simulators (where there are typically no vestibular cues) than when they normally drive. As a result, the use of GVS in static simulators may actually improve the validity of measures of driving performance.

The primary purpose of this study was to examine whether administering GVS while drivers negotiated a simulated curve in a fixed-base driving simulator would reduce SAS. We hypothesized that the application of GVS would reduce post-drive SAS (as measured by SSQ scores) through the provision of an improved impression of lateral acceleration during curved driving. The secondary purpose of this study was to compare the effect of visual cues (lamp posts) and vestibular cues (from GVS) on driving behaviours, as shown by the pattern of speed reductions and steering variability during simulated curves. We hypothesized that when both visual and vestibular cues are available, to estimate vehicular speed, drivers will reduce their speed when negotiating a curve. This in turn will improve the driver's control of the vehicle, as indicated by reduced lane deviation and steering variability. 


\section{METHODS}

\section{Participants}

Nineteen participants volunteered for the study (9 male and 10 female). Participants were intially screened for participation in the study using a general health questionnaire and motion sickness history questionnaire. For ethical reasons, those with extremely high risk of motion sickness had to be excluded from the study.

\section{Apparatus}

A Drive Safety DS-600c fixed-base driving simulator was used in testing. This simulator consisted of a Saturn four-door sedan equipped with all standard vehicle controls, augmented with audio and vibration transducers and force feedback to provide a reasonably realistic driving experience. Image generation computers projected the simulation through LCD display systems onto six, seven-foot projection screens that provided a $300^{\circ}$ wrap-around virtual environment $\left(250^{\circ}\right.$ in front and $50^{\circ}$ in the rear). Galvanic vestibular stimulation was applied using an A395 Linear Stimulus Isolator capable of producing a current output of $10 \mathrm{~mA}$. Current output during testing was adjusted to each participant's threshold to the stimulus, at a range of 0.6-1.25 mA.

The virtual environment was designed to simulate driving through a rural landscape on a sunny day. The simulated road represented a paved surface with a single lane each way and no median. Each lane was 3.6-m wide with a 1.8-m hard shoulder transitioning into a $1.8-\mathrm{m}$ dirt shoulder. In all conditions the drive consisted of 16 turns separated by $1000-\mathrm{m}$ straight road sections. The turns consisted of either a gradual $90^{\circ}$ turn or a sharp $90^{\circ}$ turn. The gradual turns consisted of a 70-m lead-in, a 314-m long curve, and a 70-m lead-out. The sharp turns were modeled on a "T" intersection and consisted of a 40-m lead-in with a 26.4-m long curve immediately transitioning back into the next roadway. The gradual and sharp turns to the left and right were presented in randomized order. In the CONTROL condition the scenery consisted of a bare rural landscape with fields and an occasional farm house. In the VISUAL ONLY and VISUAL and GVS conditions, lampposts were added to give increased visual motion cues to the participants. In the sharp turns, five posts (spaced $10 \mathrm{~m}$ apart) were placed in the lead-in area on the opposite side of the road as the turn. In addition, five posts $(10 \mathrm{~m}$ apart $)$ were placed across the back of the $\mathrm{T}$ intersection. In the gradual curves, all posts were placed on the opposite side of the road to the direction of the turn. In the lead-in, four posts were placed $17.5 \mathrm{~m}$ apart, during the curve 20 posts were placed $15 \mathrm{~m}$ apart, and during the lead-out four posts were placed $17.5 \mathrm{~m}$ apart.

\section{Experimental procedure}

Each participant drove two virtual driving worlds (with a 15-minute break in between) on two separate days. Test days were scheduled $48 \mathrm{hrs}$ apart. Over the two days, participants drove in each of the virtual driving worlds, CONTROL, GVS ONLY, VISUAL ONLY, VISUAL and GVS (counterbalanced between participants). In the GVS conditions, stimulation was applied during gradual and sharp curves for one of the drives on each day, with the order counterbalanced across participants. During GVS trials, stimulation was applied when drivers reached the end of the lead-in and entered the turn. GVS was deactivated when the vehicle 
reached the end of the turn and entered the lead-out. After each drive, participants exited the vehicle and returned to the waiting area, where they filled out their responses to the SSQ.

\section{Data Analysis}

To evaluate our first hypothesis, that application of GVS decreases SSQ scores, a two-tailed Wilcoxon signed rank analysis was used to compare Total SSQ score and sub-scale scores of Nausea, Oculomotor, and Disorientation between the CONTROL drive and GVS drive. Nonparametric analysis of the SSQ scores was required as inter-subject variability of responses was considerable (range 0 to139). As such, each participants score was coded numerically as a 1 (scores $<20)$ or 2 (scores $>20)$. A score of 20 was chosen as the division based on the work of Kennedy et al. (1993), who reportd that an SSQ score of 15 was the mean population score in response to VE simulators. Thus scores under 20 were considered to be equal to the severity of symptoms typically experienced by users of VE simulators, while scores greater than 20 reflected symptom severity greater than the population mean score and thus were indicative of becoming "sick." To evaluate the second hypothesis, whether the combination of visual and vestibular cues would improve speed estimation and vehicular control, a two-tailed Wilcoxon signed rank analysis was performed on the driving variables: Steering control, Lane departures and Mean speed. Comparisons of variables were made for each driving condition (total 4: CONTROL, VISUAL ONLY, GVS ONLY, VISUAL and GVS). Analyses of driving performance were conducted on measures taken while driving the gradual curves only, as this was the focus of our research questions.

\section{RESULTS}

\section{SSQ Scores}

Application of GVS while driving curves significantly reduced post-driving Total SSQ scores, and Disorientation sub-scores (Figure 1). No significant differences were observed between CONTROL and GVS for reported Nausea and Oculomotor sub-scores.

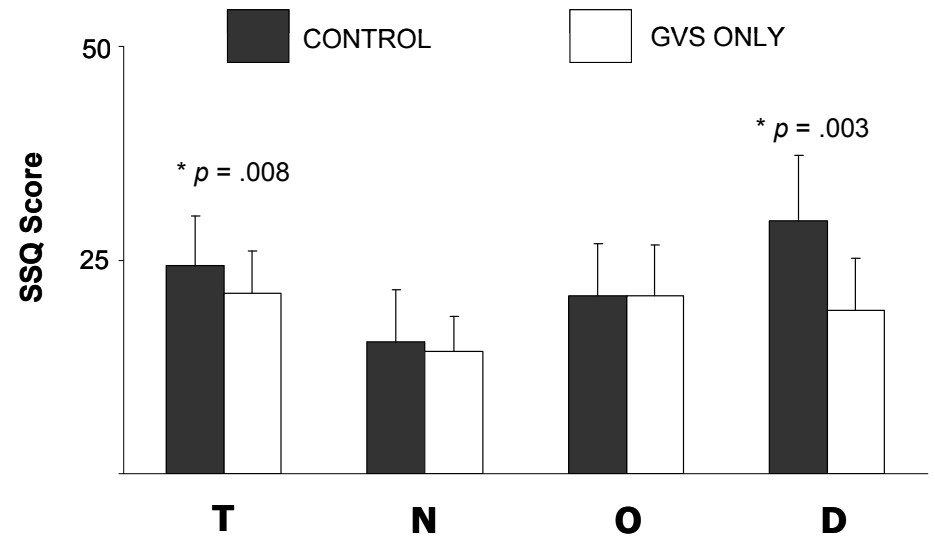

Figure 1. Mean and standard error of post drive SSQ scores 


\section{Driving performance}

Steering variability. Steering variability while driving curves was significantly reduced from CONTROL in the VISUAL and GVS condition $(p=.023)$. Reductions in steering variability from CONTROL were also observed in the VISUAL ONLY and GVS ONLY conditions; however, this finding was not statistically significant.

Table 1. Mean and standard deviation (in brackets) of steering deviations Driving Condition

\begin{tabular}{lccc}
\hline CONTROL & VISUAL ONLY & GVS ONLY & VISUAL and GVS \\
$3.43(1.52) *$ & $3.06(0.48)$ & $3.09(0.45)$ & $2.96(0.26) *$ \\
\hline$* p<.05$ & & &
\end{tabular}

Lane Departures. A significantly greater number of left-lane departures were made in the VISUAL ONLY condition regardless of curve direction $(p=.036)$. In addition, a significantly greater amount of time was spent partially out of the lane while driving curves in the VISUAL ONLY condition $(p=.033)$.

Average Speed. While no statistically significant differences were observed for average vehicular speed while negotiating curves, there were interesting trends in speed reduction, as shown in Figure 2. During the VISUAL and GVS condition and the VISUAL ONLY condition, the pattern of speed reduction from the lead-in area to the curve itself resembled what one would expect in real-world or motion-platform driving. This pattern was not seen in the CONTROL and GVS ONLY conditions.

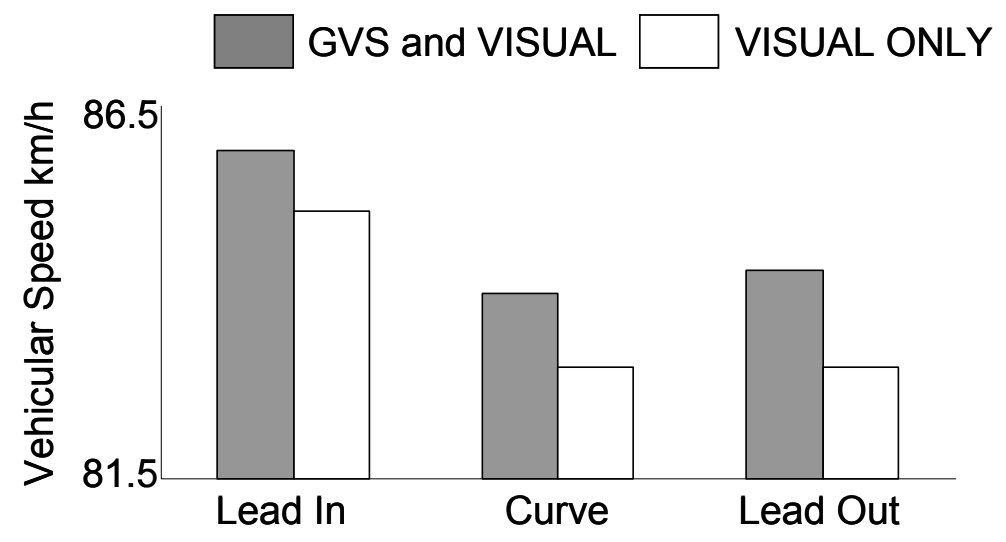

Figure 2. Mean vehicular speeds during curved driving for each driving condition

\section{DISCUSSION}

This study is the first direct evaluation of the effects of GVS on Simulator Adaptation Syndrome (SAS) as applied in a fixed-based (static) driving simulator. Reductions in SSQ scores due to the application of GVS while negotiating curves were observed. This suggests that, as hypothesized, 
the application of GVS during simulated driving can help reduce symptoms of SAS. Not only did GVS reduce overall symptoms of SAS (as measured by Total SSQ), but GVS significantly reduced Disorientation (D) symptoms (Figure 1). Typically, in static VE simulators Disorientation scores (D) are greater than Oculomotor discomfort scores $(\mathrm{O})$, which are in turn greater than Nausea scores (N). However, the pattern observed for the GVS ONLY condition was the same as in dynamic VE simulators (Jaegar \& Mourant, 2001), with $\mathrm{O}>\mathrm{D}>\mathrm{N}$. These results support the contention that GVS may be an effective alternative to motion-base platforms in simulating lateral motion.

Our hypothesis, that by providing both visual and vestibular velocity cues greater vehicular control would be observed, was supported. Significantly less steering variability was observed when both visual cues and GVS were applied. Of particular interest is that steering deviations were not significantly reduced by visual cues alone (Table 1). This observation supports observations of previously published research that vestibular cues are critical for vehicular control in curve driving (Reymond et al., 2001). GVS had no effect on lane departures. However, there was a problem with the lane deviation measure in this study as drivers consistently deviated to the left when making turns. Although the presence of lampposts next to the road may have been a factor in left-hand curves, it is unclear why this happened in right-hand curves.

In conclusion, GVS seems to be a promising intervention for reducing SAS in static driving simulators. In addition, we have observed evidence that GVS may also promote more realistic driving behaviour when negotiating curves. Nonetheless, more research on this topic is necessary, as SAS continues to be a leading methodological problem when driving performance is assessed in virtual environments. This study was the first in a larger project examining the use of GVS in fixed-base driving simulators.

\section{ACKNOWLEDGMENTS}

We would like thank David Wilson, Dana Lemoine, Lauren Meegan and Ryan Toxopeus. We would also like to acknowledge the financial support of the Canada Foundation for Innovation, the Ontario Innovation Trust, AUTO21 Network of Centres of Excellence, and the Ministry of Training, Colleges and Universities Ontario Graduate Scholarship (RRJ).

\section{REFERENCES}

Fitzpatrick, R., \& Day, B. (2004). Probing the human vestibular system with galvanic vestibular stimulation. Journal Applied Physiology, 96, 2301-2316.

Flanagan, M., May, J., Dobie, T. (2004). The role of vection, eye movements and postural instability in the etiology of motion sickness. Journal of Vestibular Research, 14, 335-346.

Jaeger, B.K., \& Mourant, R.R. (2001). Comparison of simulator sickness using static and dynamic walking simulators Proceedings from the Human Factors and Ergonomics Society 45th Annual Meeting.

Kemeny, A., \& Panerai, F. (2003). Evaluating perception in driving simulation experiments. Trends in Cognitive Sciences, 7(1), 31-37. 
Kennedy, R., Lane, N., Berbaum, K., Lilienthal, M. (1993). Simulator sickness questionnaire: an enhanced method for quantifying simulator sickness. International Journal of Aviation Psychology, 3, 203-220.

Reymond, G., Kemeny, A., Droulez, J., Berthoz, Alain. (2001). Role of lateral acceleration in curve driving: driver model and experiments on a real vehicle and a driving simulator. Human Factors: The Journal of the Human Factors and Ergonomics Society, 43(3), 483495.

Stanney, K.M., Mourant, R.R., Kennady, R.S. (1998). Human factors issues in virtual environments: a review of the literature. Presence, 7(4), 327-351. 